Introduction Patients with liver cirrhosis are immunocompromised and prone to invasive infection where outcomes are usually poor. In particular, cirrhosis is a major risk factor for severe pneumococcal infection which has a mortality rate of up to $20 \%$. Whilst patients awaiting liver transplantation routinely receive the pneumococcal vaccine, it is less clear whether the remainder of patients with cirrhosis do so or not. The present literature suggests that pneumococcal vaccination prophylaxis should be administered in cirrhotic patients and revaccination desirable at 5-yearly intervals.

The aims of this study were to assess whether patients with liver cirrhosis received pneumococcal vaccination in line with current recommendations at a single centre in the UK.

Methods A retrospective analysis of all patients with biopsy-proven liver cirrhosis, irrespective of aetiology, over a 7-year period (2005-2012) at Barnet and Chase Farm Hospitals was performed. We used a database of patients with biopsy-proven cirrhosis created by our histopathology department. Patient's primary care physicians were contacted in writing to assess whether they had received the pneumococcal vaccine.

Results 37 patients had biopsy-proven liver cirrhosis over the audit period. There was no response from the primary care physicians of 6 patients. Data for 31 patients (17 male, 14 female), median age 50 years were analysed. 14 (45\%) patients had received the pneumococcal vaccine. 4 (28.5\%) of these patients were vaccinated after their histopathological diagnosis of cirrhosis was proven. The median time interval from diagnosis to vaccination was 24 months in this group of patients. Of the 7 patients vaccinated before 2007 , none had received a repeat vaccine after 5 years.

Conclusion More than half of patients with biopsy-proven liver cirrhosis in our study did not receive the pneumococcal vaccine placing these patients at risk of life threatening preventable disease. None of the patients who received the vaccine initially were revaccinated at five yearly intervals as present literature would recommend.

We would recommend an increased awareness of the importance of regular pneumococcal vaccination to all healthcare professionals, primary care physicians and those in secondary care, who come into contact with this important and growing cohort of patients.

Disclosure of Interest None Declared.

\section{PTU-138 POPULATION-BASED STUDY OF ETHNICITY AND THE DIAGNOSIS GAP IN LIVER DISEASE}

W Alazawi ${ }^{*}$, R Mathur, K Abeysekera, S Hull, K Boomla, J Robson, GR Foster. The Blizard Institute, Queen Mary, University of London, London, UK

\subsection{6/gutjnl-2014-307263.212}

Introduction Awareness of liver disease as a major cause of morbidity and mortality has led to an increase in liver function tests (LFTs) performed in primary care with abnormal results a common finding. However, we hypothesise that a large gap exists between numbers of patients with abnormal LFTs and those with recorded liver diagnoses. Non-alcoholic fatty liver disease (NAFLD) is the most common cause of chronic liver injury. Metabolic syndrome, common in people from the Indian subcontinent, is an important risk factor for NAFLD. We hypothesise that NAFLD is more common among adults of South Asian ethnicities.

Methods In a cross-sectional study of 690,683 adults, registered in co-terminus general practices in a region with high ethnic diversity, we extracted demographic information and clinical care data including LFTs in the previous two years, liver disease diagnoses and co-morbidites from the EMIS Web computerised medical records. The breakdown of age, gender, deprivation, BMI, smoking status, alcohol consumption, co-morbidities and cholesterol was described for the whole population and for the six main ethnic groups of Bangladeshi, Indian, Pakistani, White, African and Caribbean. STATA 12 was used to conduct multivariate logistic regression analyses.

Results LFTs were performed on 218,032 patients, of whom 31,627 had elevated serum transaminases. Testing varied by age, ethnicity and the presence of co-morbidities. The prevalence of abnormal LFTs was highest among Bangladeshis and independent risk factors for abnormal LFTs included male gender, alcohol consumption and elements of the metabolic syndrome.

The most commonly recorded liver diagnosis was NAFLD, followed by chronic viral infection and alcoholic liver disease. $88.4 \%$ ( $\mathrm{n}=27,985$ ) of patients with abnormal LFTs did not have a coded liver diagnosis.

The prevalence of recorded NAFLD was highest among patients of Bangladeshi ethnicity. In a multivariate analysis, independent risk factors for NAFLD included Bangladeshi ethnicity, diabetes, raised BMI, hypertension and hypercholesterolaemia.

Conclusion Abnormal LFTs are common in the population, but are under-investigated and often remain undiagnosed. Bangladeshi ethnicity is an important independent risk factor for NAFLD. Among the group of patients with abnormal LFTs and no recorded liver diagnosis, many will have a liver disease that is amenable to further management, which may prevent complications. There is a need for evidence-based guidelines for the investigation, referral and management of patients with abnormal liver tests in the community in order to ensure early identification of treatable disease.

Disclosure of Interest None Declared.

\section{PTU-139 OUTCOMES OF PATIENTS WITH LIVER CIRRHOSIS IN A NON LIVER-SPECIALIST INTENSIVE CARE UNIT: DO ADMISSION LACTATE AND APACHE 2 SCORE HELP PREDICT SUCCESSFUL DISCHARGE?}

W Waddingham*, M Cepkova. Intensive Care, Whittington Health, London, UK

\subsection{6/gutjnl-2014-307263.213}

Introduction Hospitalised patients with cirrhosis often require admission to intensive care (ITU), usually for management of secondary complications such as GI bleeding, sepsis, or organ failure. Prognosis of these patients is poor. Despite demonstration of modest improvements in recent years, overall in-hospital mortality in this group remained $54.6 \%$ in a recent study. ${ }^{1}$ Our study aimed to explore the characteristics and outcomes of cirrhotic patients admitted to ITU, and whether this was comparable to published data. We also aimed to explore whether parameters such as APACHE 2 score and admission blood lactate differ between those who survived their ITU stay and those that did not, and if this may help predict discharge.

Methods A retrospective analysis was performed of patients admitted to the Whittington hospital ITU from January 2011June 2013. Information regarding patients with a diagnosis of cirrhosis was gathered from the Intensive Care National Audit and Research unit (ICNARC) database, and discharge summaries.

Results We identified 60 patients with cirrhosis, 3.07\% of total ITU admissions, mean age 54.8 years (range 19-78). 49/60 